\title{
Photometric and kinematic properties of disky elliptical galaxies
}

\author{
Cecilia Scorza. Landessternwarte-Königstuhl, \\ D-W6900 Heidelberg, Germany
}

New insights in the structure of elliptical galaxies have been obtained indicating the presence of faint stellar disks in some of these systems. Clear correlations between the isophotal shapes, the kinematic, radio and $\mathrm{X}$-ray properties of disky E's were found, suggesting that these galaxies form together with SO's a continuous transition of $D / B$ ratio in the Hubble sequence (Bender et al. 1989). The isophotal shapes of these galaxies have been quantified by the fourth cosine coefficient $a_{4}$ of the Fourier expansion of the deviations from perfect ellipses, which yields positive values when the isophotes are pointed along the major axis (Carter 1987, Lauer 1985, Jedrzewski 1987, Bender and Möllenhoff 1987).

Based on a photometric decomposition, we were able to disentangle, in a first aproximation, the kinematic curves of a sample of disky E's into the corresponding rotation and velocity dispersion curves of their disk and bulge components. This decomposition turns out to be an important task to test the continuity of physical properties towards lower $\mathrm{D} / \mathrm{B}$ ratios in the Hubble sequence, and hence to investigate common formation processes with $\mathrm{SO}$ galaxies.

The photometric decomposition consists in the iteractive subtraction of a sum of exponential or/and ring-type thin disk models from the original frames. The disk-model parameters are free. They are successively varied until a bulge having perfectly elliptical isophotes remains. In contrast with other decomposition methods, in this new approach special emphasis has been made on the disk modelling, because in this way the inclination of the disk (and of the galaxy) can be constrained, which is of interest for dynamical models. A first version of this method was limited to single exponential thin disk-models (for details see Scorza and Bender 1990).

The kinematic decomposition applied here relies on the fact that the line-of-sight velocity distributions (LOSVDs) of many disky ellipticals are asymmetric. These are interpreted as the superposition of two distinct kinematical components: a rapidly rotating cold component (a disk) and a more slowly rotating hot component (a bulge). The developement of new methods for kinematic analysis had made possible the detection of these asymmetries (Franx and Illinworth 1988, Bender 1990, Rix and White 1992). In a first aproximation, the observed LOSVD can be described by assuming that the line profiles of both components are gaussians. A double gaussian fit to the LOSVD of the spectral lines is then performed under the constraint that the ratio of the integrated flux of the two gaussians is equal to the 
local D/B ratio, as derived from the photometry.

The kinematic decomposition is ilustrated here for the case of NGC 3610. The photometric decomposition of this galaxy has been described with detail in Scorza and Bender (1990). Shown in Fig. 1.a is the LOSVD of the major axis spectra at $3.5,5.5,8,11$ arcsec from the center of the galaxy (solid line), the gaussians corresponding to the disk and bulge components (dotted lines) and the fit to the LOSVD (dashed line). The $\mathrm{D} / \mathrm{B}$ ratio values used to constrain the fit are given in each panel. The quality of the fit indicates that the shape of the LOSVD of NGC 3610 can be undestood within the simple two-component picture. Once the fit has been made, the rotation and velocity dispersion curves of the disk and bulge can be obtained. These are shown in Fig. 1.b together with the kinematical curves derived in the conventinal way from a single-Gauss fit to the LOSVD (see small vs. big symbols). The $v / \sigma=3.4$ of the disk corresponds to a cold stellar component. This result is in good agreement with that obtained by Rix and White (1992). The $(v / \sigma)^{*}=1.13$ of the bulge indicates that it is rotationally flattened. From ten disky E's examined so far, eight have rotationally flattened bulges and cold inner disks. More details and discussion are given in Scorza 1992.

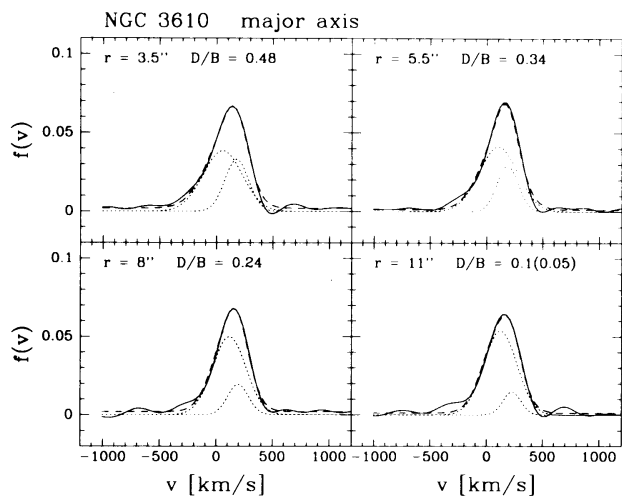

Fig. 1.a

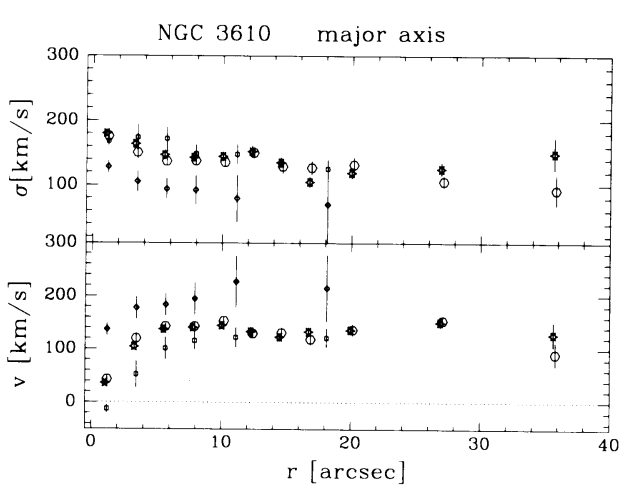

Fig. 1.b

Bender, R., Möllenhoff, C.: 1987, Astron. Astrophys. 177, 71

Bender, R.: 1988, Astron. Astrophys. Letters L193, L7

Bender, R.: 1990, Astron. Astrophys. 229, 441

Carter, D.: 1987, Astrophys. J. 312, 514

Franx, M., Illingworth, G.D: Astrophys. J. , 344L55,

Jedrzewski, R.I.: 1987, Monthly Notices Roy. Astron. Soc. 226, 747

Lauer, T.R.: 1985, Monthly Notices Roy. Astron. Soc. 216, 429

Nieto, J-L., Bender, R., Surma, P. : 1991, Astron. Astrophys. Letters L244, L37

Rix, H-W., White, S.: 1992, Monthly Notices Roy. Astron. Soc. 254, 389

Scorza, C., Bender, R.: 1990, Astron. Astrophys. 235, 49

Scorza, C. : 1992. Ph.D. Thesis, Univ. of Heidelberg. 